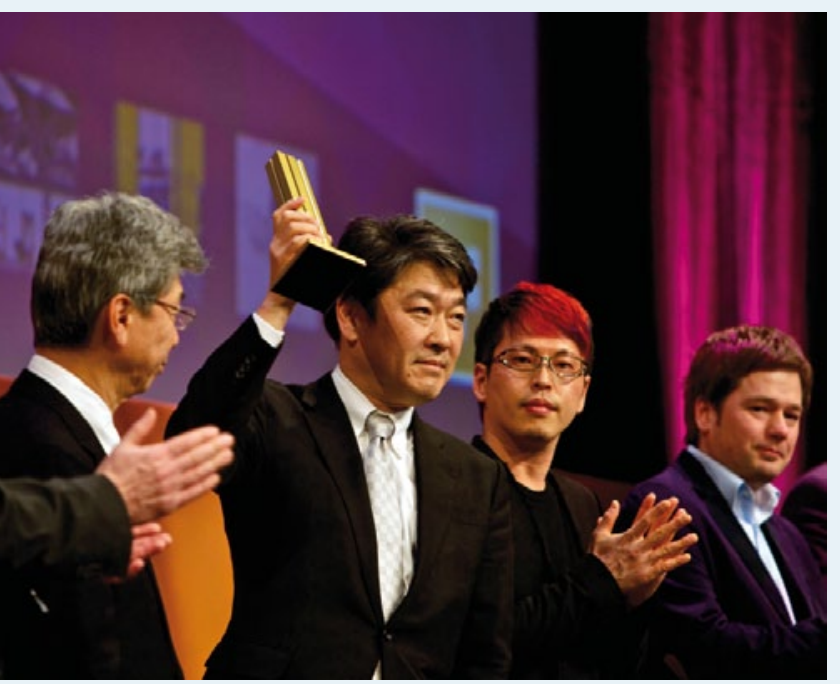

Die gemeinsam mit der Münchner Design-Agentur f/p design GmbH gestaltete Behandlungseinheit Soaric erhielt für das Produktdesign die höchste Auszeichnung bei der iF design award night 2012, den iF gold award. Zudem wurde die japanischdeutsche Gemeinschaftsarbeit in der Kategorie communication design für die
Bedienoberfläche sowie für die Gestaltung von Broschüre und Werbeanzeige mit weiteren $2 \mathrm{iF}$ awards geehrt.

Erstmals zieht Morita nach der Verleihung in das iF-Design-ranking ein. Stolz und zufrieden präsentierten die beiden japanischen Präsidenten die Trophäen nach der Preisübergabe. Für das innovative Familienunternehmen waren gleich mehrere Verantwortliche extra aus Japan angereist, um die international anerkannten und bedeutenden Design-Auszeichnungen entgegenzunehmen.

Der bereits im März 2011 auf der Internationalen Dental-Schau (IDS) in Köln der Öffentlichkeit vorgestellte Arbeitsplatz vereint eine Funktionalität mit einer nun ausgezeichneten Ästhetik. Bei der Gestaltung des Arbeitsplatzes wurden besonders die Anforderungen an Bewegungsräume für Endodontie-Behandlungen berücksichtigt. Alle Instrumente sind so angeordnet, dass sie eine 4-Hand- oder 6-Hand-Behandlung ideal unterstützen und situationsgerechtes Anreichen ermöglichen. Auch das integrierbare Operationsmikroskop mit vernetztem Monitor sowie das bedienfreundliche Interface des LC-Touch-Displays im Arztelement und der Rückzugsentlastungsmechanismus der Instrumentenschläuche tragen zum Behandlungskomfort bei. Die Materialien der Einheit, die klare Designstruktur und intuitiv zu bedienende Arbeitselemente überzeugten bislang Zahnärzte, die an der Einheit behandeln - und nun auch die iF-Jury.

Das japanische Familienunternehmen Morita und die Münchner Designagentur f/p design wollen die erfolgreiche Zusammenarbeit auch in Zukunft weiterführen: Am Rande der Preisverleihung wurde bekannt, dass die Kooperation schon weitere preisverdächtige Früchte trägt.

Nach einer Pressemitteilung der

J. Morita Europe $\mathbf{G m b H}$, Dietzenbach

Internet: www.morita.com/europe

\title{
Bonding auf den Punkt gebracht
}

Das unabhängige amerikanische Testinstitut „The Dental Advisor" bestätigte die hohe Zuverlässigkeit und Qualität der iBOND ${ }^{\circledR}$ _ Produkte zum Jahresbeginn 2012 gleich doppelt: Das All-in-One-Adhäsiv iBOND ${ }^{\circledR}$ Self Etch wurde mit der Bestnote von 5 Plus mit dem „Editors“ Choice Product Award“ ausgezeichnet. Die-

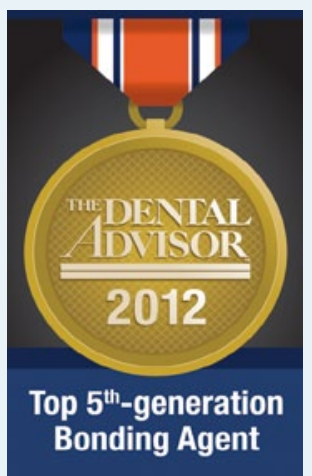
bondet und desensibilisiert. Es kombiniert hohe Haftfestigkeit und optimale Randqualität mit einfacher, schneller und anwenderfreundlicher Applikation. Ein verbesserter Tropfer erlaubt eine präzise Dosierung und vermeidet das Verkleben der Flasche. Das Material eignet sich für die Befestigung direkter lichthärtender Kompositwerksen renommierten Preis erhielt mit insgesamt 4,5 Bewertungspunkten auch das neue Etch \& Rinse 2-StepAdhäsiv iBOND ${ }^{\circledR}$ Total Etch, das zudem in die Liste der „Preferred Products 2012“ aufgenommen und als „TOP 5th Generation Bonding Agent" ausgezeichnet wurde.

iBOND $^{\circledR}$ Self Etch ist ein lichthärtendes selbstkonditionierendes Einkomponenten-Adhäsiv der 7. Generation, das in einem einzigen Arbeitsschritt ätzt, primt, stoffe, indirekter Restaurationen in Kombination mit einem lichthärtendem Befestigungskomposit und für die Behandlung überempfindlicher Zahnbereiche.

Das lichthärtende Etch \& Rinse 2-StepAdhäsiv iBOND ${ }^{\circledR}$ Total Etch ist das neue Bonding-System der 5. Generation. Nach dem Ätzvorgang wird es in nur 1 Schicht appliziert, die nicht aufwendig einmassiert werden muss. Das auf Nanofüllern basierende Gesamtsystem erzielt einen perfekten Randschluss und hohe Haftwerte an Schmelz und Dentin und ist darüber hinaus ideal für die Desensibilisierung von hypersensitiven Bereichen geeignet. Die idealen Filmbildungseigenschaften gewährleisten hohe Anwendersicherheit, das punktgenaue kontrollierte Dosieren und die Lagerung bei Raumtemperatur vereinfachen das Handling. Das Adhäsiv ist für das Befestigen von direkten Kompositrestaurationen, von indirekten laborgefertigten Restaurationen und für die Behandlung überempfindlicher Zahnbereiche indiziert.

Beide Produkte werden in der Mai-Ausgabe 2012 des Dental Advisors ausführlich vorgestellt. Die vollständigen Bewertungen der Product Awards sowie der Preferred Products 2012 können unter www.dentaladvisor.com abgerufen werden.

Nach einer Pressemitteilung der Heraeus Kulzer GmbH, Hanau Internet: www.heraeus-dental.com 


\section{„Wir können als Marke selbstbewusst auftreten“}

Interview mit Frank Bartsch, Trade Marketing Manager Carestream Dental (rechts außen), und Nicola Gizzi, Vertriebsleiter Carestream Dental, über den Marken-Switch von Kodak Dental Systems zu Carestream Dental.

\section{? Kodak Dental Systems heißt jetzt Carestream Dental. Wie bekannt ist die Marke Carestream Dental in der Zahn- medizin?}

Bartsch: Carestream Dental ist als Marke bei den Zahnärzten natürlich noch nicht so bekannt wie Kodak, die es ja schon hundert Jahre gibt. Das ist ein dynamischer Prozess, an dem wir bereits seit einigen Jahren intensiv arbeiten, und der weiterhin Zeit in Anspruch nehmen wird. Zahnmediziner, die sich für Kodak Dental Systems interessieren, finden diese seit 2007 an den Messeständen von Carestream Health und seit 2010 bei Carestream Dental. Der Name Carestream steht damit für hochqualitative Spezialprodukte.

Gizzi: Darauf aufbauend tragen unsere Neuprodukte seit der IDS 2011 nicht mehr das Label Kodak Dental Systems, sondern CS als Abkürzung für Carestream. Unter anderem haben wir so das DVT CS 9300 oder den Intraoralscanner CS 7600 im Jahr 2011 in den Markt eingeführt. Lediglich die Röntgenfilme sind weiter unter dem Label Kodak Dental Systems erhältlich. Produzent und Inhaber der Patente ist aber Carestream Dental.

\section{? Wie ist die Markteinführung der CS-Produkte verlaufen?}

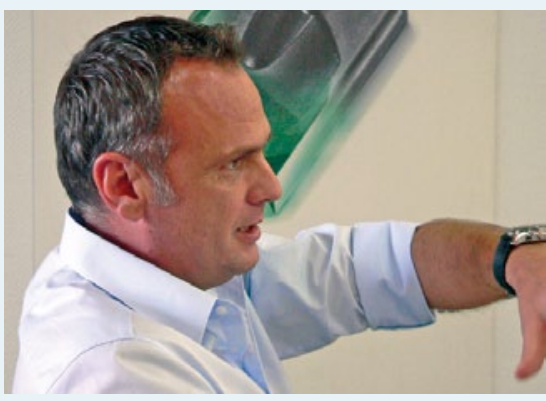

Gizzi: Der Name allein hat die Nachfrage nicht bestimmt, sondern die Features der Produkte. Wir haben auf der IDS und auch auf den regionalen Fachmessen 2011 verschiedene Innovationen vorgestellt und uns klar als Imaging Spezialist positioniert. Unser neues DVT CS 9300 bietet durch sein „Flexi-Field-of-View“ von $5 \times 5-17 \times 13,5 \mathrm{~cm}$ alle für die Zahnarztpraxis notwendigen Volumengrößen, sodass durch Auswahl des optimalen Volumens die Strahlendosis so gering wie möglich gehalten werden kann. Mit dieser und weiteren Funktionen hat das CS 9300 im Markt eine hohe Akzeptanz erreicht.

\section{? Was bedeutet der Marken-Switch} für den technischen Service?

Gizzi: Die Geräte mit dem Label Kodak Dental Systems stammen aus der Entwicklung von Carestream. Sie wurden in dem gleichen Werk nahe Paris produziert, in dem auch die Carestream Dental Produkte hergestellt werden. Bei der Ersatzteilbeschaffung und dem technischen Service wird es also keine Veränderungen geben, da sich weder die Produktions-
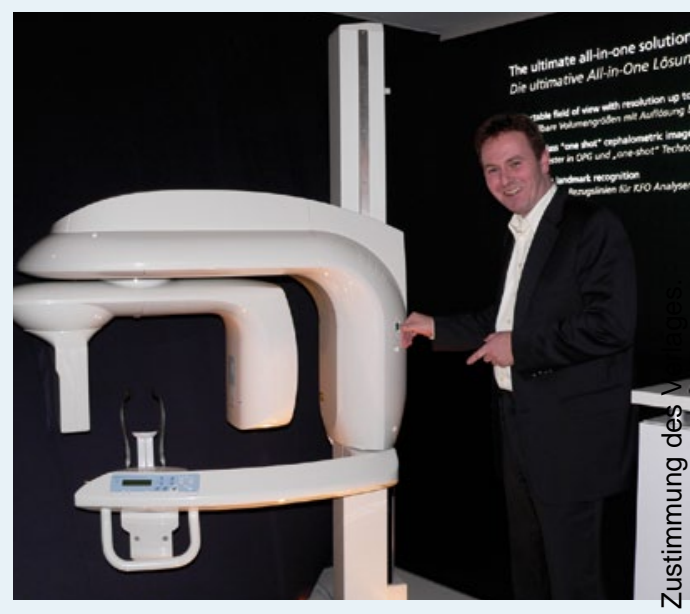

noch die Unternehmensstruktur verändern.

? Können Sie schon konkret sagen, welche Entwicklungen im Fokus stehen? Bartsch: Während des Kick-Off-Events in Sonthofen haben wir unter dem Siegel der Verschwiegenheit den Prototyp eines neuen Röntgengerätes von Carestream Dental präsentiert. Die Meinung unserer Händler zu diesem neuen Produkt war uns sehr wichtig und hat uns darin bestätigt, dass wir mit dieser Innovation auf einem sehr guten Weg sind. Ich bin mir sicher, dass die Fachwelt von dem neuen Gerät begeistert sein wird. Die offizielle Produktpräsentation ist für Sommer 2012 geplant. Es ist also noch etwas Geduld gefragt, bevor ich Ihnen mehr verraten kann!

Nach einer Pressemitteilung der Carestream Health Deutschland GmbH, Stuttgart Internet: www.carestreamdental.com

\section{Eiskalt erfrischter Atem und sofort* weißere Zähne}

Mit Ice Cool Mint führt Signal eine neue Geschmacksrichtung der WhiteningZahnpasta White Now ein. Sofort nach der Anwendung mit White Now Ice Cool Mint wirken die Zähne weißer und der Atem ist eiskalt erfrischt. Die UnileverForschung entwickelte mit der neuen Zahnpasta das 1. Whitening-Produkt, das seine Wirkung bereits nach einmaliger Anwendung entfaltet. Möglich macht das die Formel mit Active Blue Foam. Der Wirkstoff Blue Covarine legt sich auf die Oberfläche des Zahnes und verändert dessen optische Eigenschaften. Der Gelbstich der Zähne wird gemindert, die Zähne erscheinen sofort weißer und glänzender. Das Produkt unterscheidet sich damit von allen Whitening-Zahnpasten, die auf Basis von Bleaching oder Abrasion arbeiten. Die neue Geschmacksrichtung sorgt für eiskalt erfrischten Atem. Mit einem Fluoridgehalt von $1450 \mathrm{ppm}$ und einem geringen Abrasionswert von ca. 85 ist die

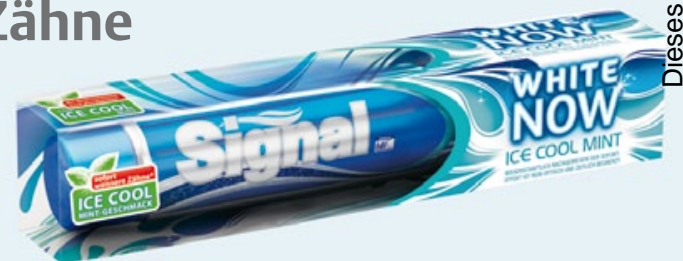

Zahnpasta für die tägliche Anwendung geeignet.

* Der Soforteffekt ist rein optisch und zeitlich begrenzt.

Nach einer Pressemitteilung der Unilever Deutschland GmbH, Hamburg

Internet: www.signal-zahnpflege.de 


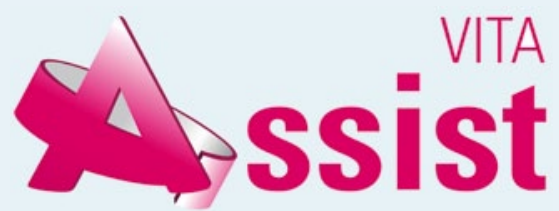

Im dentalen Bereich sind moderne computergestützte CAD/CAM-Fertigungstechniken und digitale Planungsmöglichkeiten auf dem Vormarsch. Die neue Software VITA Assist von der VITA Zahnfabrik unterstützt die Kommunikation. Die Software ist für $299 €$ seit Februar erhältlich. Sie optimiert die Kommunikation zwischen Zahnarzt und Zahntechniker als auch zwischen Behandler und Patient, indem sie eine noch schnellere und umfassendere Datenverwaltung ermöglicht.

Mithilfe des Programms ist der Zahnarzt/ Zahntechniker jederzeit in der Lage, Patientendaten, zahnmedizinische Befunde und digitale Fotos zu erfassen, zu bearbeiten und zu verwalten und diese anschließend - per Knopfdruck - als E-Mail oder Ausdruck weiterzuleiten. Auch die Dokumentation von Zahnfarbbestimmungen

\section{viele Möglichkeiten}

und Bleaching-Prozessen sowie die interaktive Simulation von Voll- und Teilprothesen sind Stärken des Programms. Um alle diese Features mit einem einzigen Programm abdecken zu können, hat das Unternehmen die Module VITA ShadeAssist und VITA ToothConfigurator unter dem neuen Programm vereint.

VITA ShadeAssist garantiert eine verlustfreie Übermittlung und Verwaltung aller Zahnfarbinformationen, die für eine Reproduktion notwendig sind, unabhängig davon, ob die Farbbestimmung mit dem VITA Easyshade oder einer VITA Farbskala erfolgt. Der VITA ToothConfigurator gibt Patienten die Möglichkeit, ihr individuelles prothetisches Ergebnis aktiv mitzugestalten und sich bereits in der Planungsphase „ein Bild“ von dem neuen Zahnersatz zu machen.

Nach einer Pressemitteilung der

VITA Zahnfabrik H. Rauter GmbH \& Co. KG, Bad Säckingen

Internet: www.vita-zahnfabrik.com
Kaltpolymerisierendes Komposit

Einfach, ästhetisch, stabil

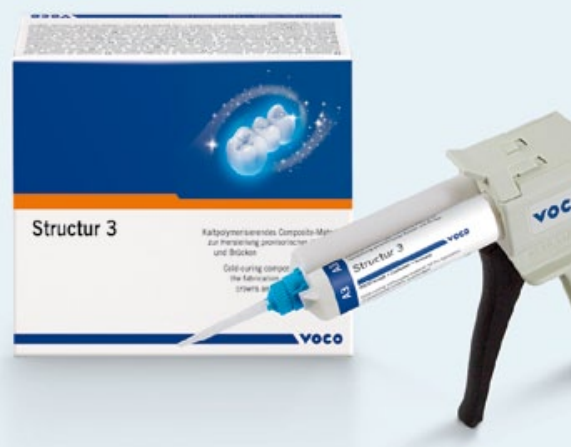

Structur 3 ist das neue selbsthärtende K\&B-Material von VOCO. Es dient zur schnellen Herstellung von qualitativ hochwertigen provisorischen Kronen und Brücken sowie von Inlays, Onlays, Teilkronen, Veneers und Stiftprovisorien. Auch Langzeitprovisorien lassen sich mit dem Material fertigen. Eine weitere Indikation ist die Unterfütterung von vorgefertigten, provisorischen Kronen aus Komposit, Polykarbonat oder Metall. Das neue Material verfügt über eine außerordentliche Stabilität und hohe Ästhetik und lässt sich ebenso schnell wie einfach verarbeiten.

Das Komposit ist optimal in der Anwendung. Dank des 1:1-Mischsystems kann es ohne Mischfehler zeitsparend in den Abdruck appliziert werden. Die intraorale Aushärtezeit beträgt lediglich $45 \mathrm{~s}$. Im Anschluss kann das Provisorium mühelos abgenommen werden, da es in dieser Phase noch leicht elastisch ist. Von Mischbeginn an ist das neue Material innerhalb von nur $4 \mathrm{~min}$ vollständig ausgehärtet. Das Provisorium zeigt nach Entfernen der Inhibitionsschicht mit einem alkoholgetränkten Tuch sofort und ohne polieren zu müssen einen zahnähnlichen Glanz.

Mit Structur 3 gefertigte Restaurationen sind ästhetisch und kommen mit ihrem natürlichen Glanz und ihrer Fluoreszenz der definitiven Versorgung sehr nahe. Dazu trägt auch das breite Farbspektrum bei. Das neue Material ist in der Kartusche in 8 VITA-Farben erhältlich (A1, A2, A3, A3.5, B1, B3, C2, BL). Außerdem wird es in 4 Farben (A1, A2, A3, B1) in der praktischen QuickMix-Spritze angeboten.

Nach einer Pressemitteilung der

voco $\mathrm{GmbH}$, Cuxhaven

Internet: www.voco.com 


\section{Zeichnungsscharfe Abformung}

Das Anfließverhalten eines Abformmaterials an die noch restfeuchte Oberfläche ist eines der entscheidendsten Kriterien für hochpräzise Abformungen. Detaseal ${ }^{\circledR}$ hydroflow wurde exakt dafür entwickelt und zeigt eine ultimative Hydrophilie, nicht nur gleich bei der Applikation, sondern auch über die gesamte Verarbeitungszeit hinweg.

Fünf High-End-Abformsilikone mit harmonisierten Konsistenzen bilden ein komplettes System. Detaseal ${ }^{\circledR}$ hydroflow putty in 5:1-Hartkartuschen zeichnet sich durch eine softgeschmeidige Anfangskonsistenz aus. Detaseal ${ }^{\circledR}$ hydroflow heavy hat eine hohe Konsistenz und lässt sich nach einer Mundverweildauer von nur 2 min leicht entformen. In Kombination mit den beiden Korrekturmaterialien Detaseal ${ }^{\circledR}$ hydroflow lite \& -Xlite (beide in 2 Abbindevarianten: regular + fast set) eignet sich das Material sowohl für die Doppelmisch- als auch Sandwichtechnik. Die Korrekturmassen erreichen eine höchstmögliche Benetzungsfähigkeit sofort ab Mischbeginn. Das Ergebnis: zeichnungsscharfe Abformungen und die Reproduktion feinster Details, besonders in feuchten Bereichen, und eine exakte Darstellung der Präparationsgrenzen.

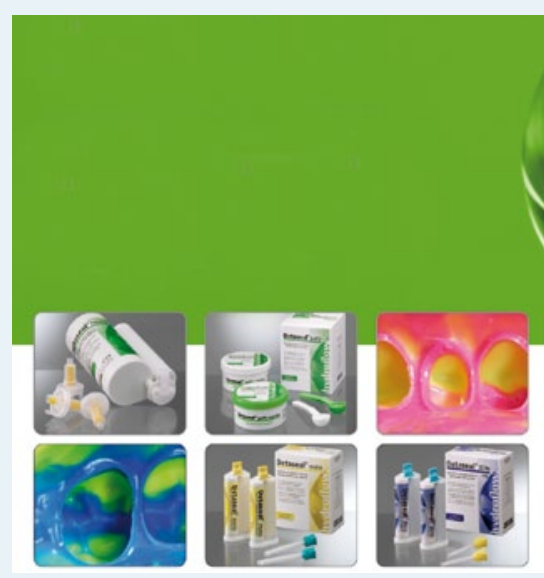

Nach einer Pressemitteilung der DETAX GmbH \& Co. KG, Ettlingen Internet: www.detax.de

\section{Auf die Größe kommt es an}

Das 2-stufige Restaurationskonzept EQUIA, aus einer hochviskösen glasionomerbasierten Füllungskomponente (EQUIA Fil) und einem hochgefüllten

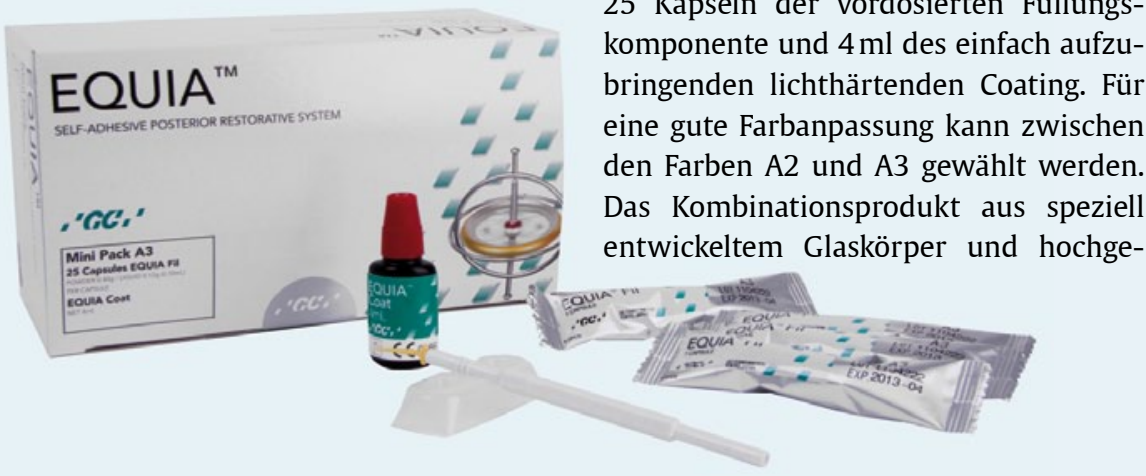

fülltem Kompositlack sorgt für hervorragende physikalische Eigenschaften. Das glasionomerbasierte Material kann auch für permanente Versorgungen bei tragenden Klasse-I-Kavitäten und kleineren kaudruckbelasteten Klasse-II-Kavitäten entsprechend den Anwendungsempfehlungen des Herstellers im Rahmen der Basisversorgung verwendet werden. Es ist schnell anwendbar und bulkapplizierbar ist.

Nach einer Pressemitteilung der GC Germany GmbH, Bad Homburg Internet: www.equia.info

\section{Für Communication Design ausgezeichnet}

Der iF communication design award 2012 geht an die Sonicare DiamondClean von Philips - das Flaggschiff der SonicareSchallzahnbürsten-Familie. Seit 1953 lädt iF internationale Hersteller und Gestalter ein, sich mit ihrem Design dem Wettbewerb zu stellen. Als „Siegel für gutes Design" sind die iF design awards in der Welt der Gestaltung und weit darüber hinaus seit Langem bekannt. Zur Jury gehörten 44 internationale Experten und renommierte Vertreter ihres Fachs. Die preisgekrönten Produkte waren vom 06.10. März 2012 auf der iF design Ausstel- lung in Hannover zu sehen und werden in der 2. Jahreshälfte in Hamburg präsentiert. Am 10. Februar 2012 fand die Preisvergabe in München statt.

„Wir arbeiten kontinuierlich daran, vom Konzept bis zur Produktion innovative Ideen zu verwirklichen. So entstehen Designs, die für Verbraucher sinnvoll und Compliance-steigernd sind“, kommentiert Sean Carney, Chief Design Officer, Philips Design.

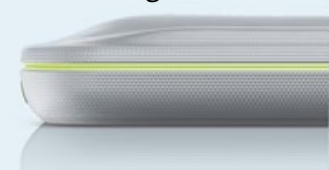

Nach einer Pressemitteilung der Philips GmbH, Hamburg Internet: www.philips.de/sonicare

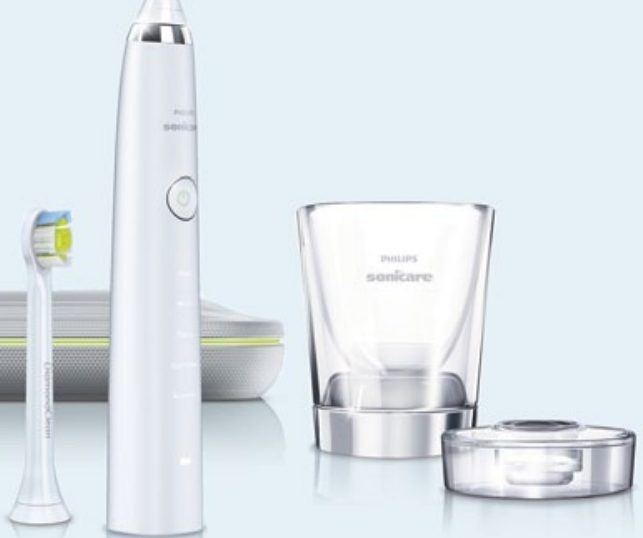


